\section{POSTHOC: A FORTRAN IV irogram \\ for computing one-way analysis of variance and post hoc Dunnett. Newman-Keuls, or Scheffé mean comparisons}

\author{
RAY T. STERNER, JAMES T. WHEELER, \\ and LAVERN F. KRABILL \\ Letterman Amy Institute of Research. \\ Presidio of San Francisco, California 94129
}

Program POSTHOC provides for an analysis of variance of data obtained with either a balanced (equal-cell) one-way independent-groups or repeated-measures design (cf. Winer, 1971), and it computes the Dunnett (cf. Dunnett, 1955), Newman-Keuls (cf. Newman, 1939; Keuls, 1952) or Scheffé (cf. Scheffé, 1953) post-hoc mean tests on these or separate data (cf. Sterner, Wheeler, \& Krabill, in press). The analysis of variance routine assumes treatments to be a fixed-effect and subjects a random-effect variable; the mean comparison routine assumes a prior significant $\mathrm{F}$ value for treatments or appropriate analysis of variance test term. The important attribute of POSTHOC is the option allowing for the separate use of three mean-comparison procedures (i.e., the user may specify the appropriate error mean square value to be used in performing selected mean comparisons).

Input. Input to the program is via punched card or magnetic tape. Missing data are treated as zeros. A free-field, F-type variable format specification provides for varied data configurations.

Output. The following output is provided: (1) A complete independent-groups or repeated-measures one-way analysis of variance source table. (2) A list of means and standard deviations for each cell of the design. (3) A matrix of actual mean differences between control and ordered treatment means (i.e., Dunnett procedure), or among all possible ordered cell means (i.e., Newman-Keuls and Scheffé procedures), for post-hoc mean comparisons. (4) A matrix of critical values for the respective mean differences, including a list of the minimum critical value(s) required for significance of each mean difference (i.e., a single value is output for the Dunnett and Scheffé procedures; $k-1$ treatment values are provided under the New man-Keuls

Present address of Ray T. Sterner: Section of Supporting Sciences, Denver Wildlife Research Center, Building 16, Federal Center, Denver, Colorado 80225.

Present address of Lavern F. Krabill: Smith, Kline \& French Laboratories, West Chester. Pennsylvania 19380. option). (5) A significance matrix in which each mean comparison exceeding the minimal Dunnett, Newman-Keuls, or Scheffé critical value(s)is marked by an asterisk.

User options. Six user-selected options for data input and statistical computations are available. These include: (1) single or multiple problem decks, (2) independent-groups or repeated-measures analysis of variance, (3) Dunnett, Newman-Keuls, or Scheffé mean comparison procedures, (4) punched card or magnetic tape input, (5) order of data according to a given subject or cell of the design, and (6) substitution of an appropriate error mean square value (obtained from external analysis of variance software) for the separate use of the mean comparison routine.

Limitations. Four limitations affect the use of POSTHOC: (1) number of treatment cells (i.e., design levels), $<40 ;(2)$ number of observations per cell, $<400$; (3) number of dependent variables per problem, 1 ; and (4) number of problems per run, dependent upon specified operation time.

Computer and Language. POSTHOC is written in FORTRAN IV for use on both CDC 6400 and 7600 hardware. 'Typical times for problems run on a 6400 machine have ranged between 6-12 sec (e.g., a sample problem having eight cell means, with 72 observations/cell, ran 6 sec and cost a total of \$1.38).

Availability. A user's write-up and program listing are available from the first author or the Department of Information Sciences, Letterman Army Institute of Research, Presidio of San Francisco, California 94129.

\section{REFERENCES}

Dunnett, C. W. A multiple comparison procedure for companing several treatments with a control. Journal of American Statistical Association, 1955, 50, 1096-1121.

Keuls, M. The use of the "studentized range" in connection with an analysis of variance. Euphytica, 1952, 1, 112-122.

Newman, D. The distribution of the range in samples from a normal population expressed in terms of an independent estimate of standard deviation. Biometrika, 1939, 31, 20-30.

Scheffé, H. A method of juding all contrasts in the analysis of variance. Biometrika, 9153, 40, 87-104.

Sterner, R. T., Wheeler, J. T., \& Krabill, L. F. Program POSTHOC: Balanced one-way independent-groups or repeated-measures analysis of variance with post-hoc Dunnett. Newman-Keuls or Scheffe mean comparisons. Letterman Army Institute of Research Laboratory Report, in press.

Winer, B. J. Statistical principles in experimental design 2 nd ed New York: McGraw-Hill, 1971. Pp. 149-168, 261-273. 